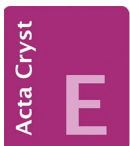

CRYSTALLOGRAPHIC COMMUNICATIONS

ISSN 2056-9890

Received 20 April 2017

Accepted 19 May 2017

Edited by E. F. C. Herdtweck, Technische Universität München, Germany

Keywords: crystal structure; $\mathrm{Ag}_{1.655} \mathrm{Co}_{1.64^{-}}$ $\mathrm{Fe}_{1.36}\left(\mathrm{PO}_{4}\right)_{3}$; transition metal phosphate; solidstate reaction synthesis; alluaudite-like structure.

CCDC reference: 1551181

Supporting information: this article has supporting information at journals.iucr.org/e

\section{Crystal structure of a silver-, cobalt- and iron-based phosphate with an alluaudite-like structure: $\mathrm{Ag}_{1.655} \mathrm{Co}_{1.64} \mathrm{Fe}_{1.36}\left(\mathrm{PO}_{4}\right)_{3}$}

\author{
Adam Bouraima, ${ }^{\mathrm{a}, \mathrm{b} *}$ Thomas Makani, ${ }^{\mathrm{b}}$ Abderrazzak Assani, ${ }^{\mathrm{a}}$ Mohamed Saadi ${ }^{\mathrm{a}}$ and \\ Lahcen El Ammari ${ }^{\mathrm{a}}$
}

\begin{abstract}
aLaboratoire de Chimie du Solide Appliquée, Faculty of Sciences, Mohammed V University in Rabat, Avenue Ibn Battouta, BP 1014, Rabat, Morocco, and ' ${ }^{\mathbf{b}}$ Département de Chimie, Faculté des Sciences, Université des Sciences et Techniques de Masuku, BP 943, Franceville, Gabon. *Correspondence e-mail: adam_bouraima@yahoo.fr
\end{abstract}

The new silver-, cobalt- and iron-based phosphate, silver cobalt iron tris(orthophosphate), $\mathrm{Ag}_{1.655} \mathrm{Co}_{1.64} \mathrm{Fe}_{1.36}\left(\mathrm{PO}_{4}\right)_{3}$, was synthesized by solid-state reactions. Its structure is isotypic to that of $\mathrm{Na}_{2} \mathrm{Co}_{2} \mathrm{Fe}\left(\mathrm{PO}_{4}\right)_{3}$, and belongs to the alluaudite family, with a partial cationic disorder, the $\mathrm{Ag}^{\mathrm{I}}$ atoms being located on an inversion centre and twofold rotation axis sites (Wyckoff positions $4 a$ and $4 e$ ), with partial occupancies of 0.885 (2) and 0.7688 (19), respectively. One of the two $\mathrm{P}$ atoms in the asymmetric unit completely fills one $4 e$ site while the Co and Fe atoms fill another $4 e$ site, with partial occupancies of 0.86 (5) and 0.14 (5), respectively. The remaining $\mathrm{Co}^{2+}$ and $\mathrm{Fe}^{3+}$ cations are distributed on a general position, $8 f$, in a 0.39 (4):0.61 (4) ratio. All $\mathrm{O}$ atoms and the other $\mathrm{P}$ atoms are in general positions. The structure is built up from zigzag chains of edge-sharing $\left[\mathrm{MO}_{6}\right](M=\mathrm{Fe} / \mathrm{Co})$ octahedra stacked parallel to [101]. These chains are linked together through $\mathrm{PO}_{4}$ tetrahedra, forming polyhedral sheets perpendicular to [010]. The resulting framework displays two types of channels running along [001], in which the $\mathrm{Ag}^{\mathrm{I}}$ atoms (coordination number eight) are located.

\section{Chemical context}

Compounds belonging to the large alluaudite structural family (Moore, 1971; Moore \& Ito, 1979; Hatert et al., 2000, 2004) have been of continuing interest owing to their open-framework architecture, with hexagonal-shaped channels, and their physical properties. This fact is amply justified by their practical applications, for example as corrosion inhibitors, passivators of metal surfaces, and catalysts (Korzenski et al., 1999). In addition, interest in alluaudite phosphates with monovalent cations has continued to grow in the electrochemical field, where they have applications as positive electrodes in lithium and sodium batteries (Trad et al., 2010). Accordingly, our attention is mostly focused on the elaboration and structural characterization of new alluaudite-type phosphates within the $A_{2} \mathrm{O}-M \mathrm{O}-\mathrm{P}_{2} \mathrm{O}_{5}$ systems $(A=$ monovalent cation $M=$ divalent cation). For instance, most recently, the hydrothermal investigation of the $\mathrm{Na}_{2} \mathrm{O}-M \mathrm{O}-\mathrm{P}_{2} \mathrm{O}_{5}$ pseudo-ternary system has allowed the isolation of the sodium- and magnesium-based alluaudite phosphate $\mathrm{NaMg}_{3}\left(\mathrm{PO}_{4}\right)\left(\mathrm{HPO}_{4}\right)_{2}$ (Ould Saleck et al., 2015). On the other hand, within the $\mathrm{Na}_{2} \mathrm{O}-\mathrm{CoO}-\mathrm{Fe}_{2} \mathrm{O}_{3}-$ $\mathrm{P}_{2} \mathrm{O}_{5}$ and $\mathrm{Na}_{2} \mathrm{O}-\mathrm{ZnO}-\mathrm{Fe}_{2} \mathrm{O}_{3}-\mathrm{P}_{2} \mathrm{O}_{5}$ pseudo-quaternary systems, solid-state synthesis has allowed $\mathrm{Na}_{2} \mathrm{Co}_{2} \mathrm{Fe}\left(\mathrm{PO}_{4}\right)_{3}$ (Bouraima et al., 2015) and $\mathrm{Na}_{1.67} \mathrm{Zn}_{1.67} \mathrm{Fe}_{1.33}\left(\mathrm{PO}_{4}\right)_{3}$ (Khmiyas et al., 2015) to be obtained. With the same objective, a new 


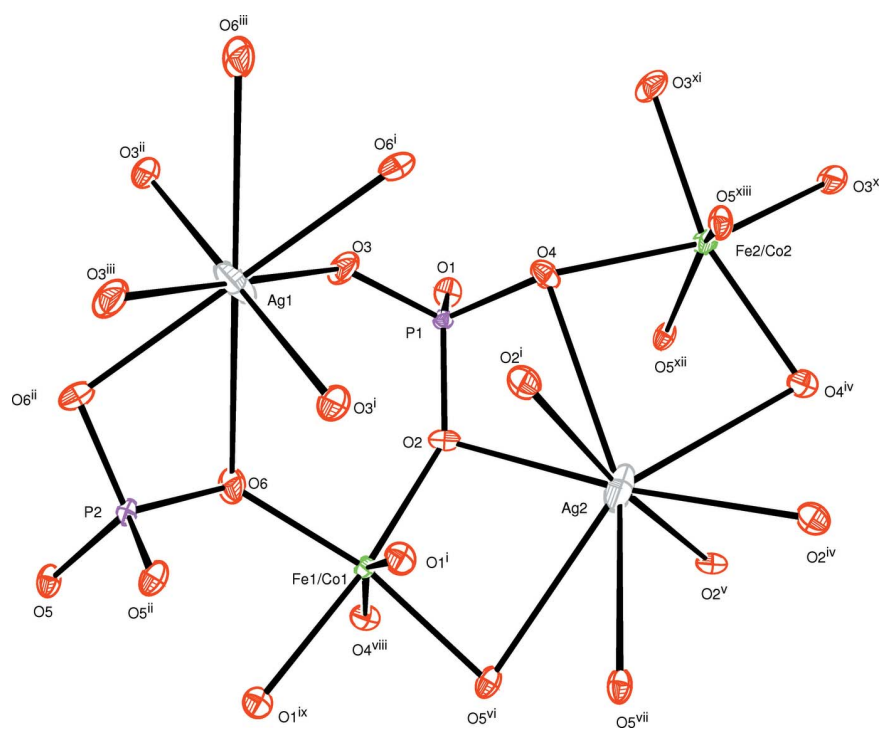

Figure 1

The principal building units in the structure of the title compound. Displacement ellipsoids are drawn at the $50 \%$ probability level. [Symmetry codes: (i) $x,-y+1, z+\frac{1}{2}$; (ii) $-x+1, y,-z+\frac{1}{2}$; (iii) $-x+1$, $-y+1,-z+1$; (iv) $-x+2, y,-z+\frac{3}{2}$; (v) $-x+2,-y+1,-z+1$; (vi) $x+\frac{1}{2}$, $-y+\frac{1}{2}, z+\frac{1}{2}$; (vii) $-x+\frac{3}{2},-y+\frac{1}{2},-z+1$; (viii) $x,-y+1, z-\frac{1}{2}$; (ix) $-x+\frac{3}{2}$, $y-\frac{1}{2},-z+\frac{1}{2} ;(x) x+\frac{1}{2},-y+\frac{3}{2}, z+\frac{1}{2}$; (xi) $-x+\frac{3}{2},-y+\frac{3}{2},-z+1$; (xii) $-x+\frac{3}{2}$, $y+\frac{1}{2},-z+\frac{1}{2}$; (xiii) $x+\frac{1}{2}, y+\frac{1}{2}, z+1$.]

silver-, cobalt- and iron-based alluaudite-type phosphate, namely $\mathrm{Ag}_{1.655} \mathrm{Co}_{1.64} \mathrm{Fe}_{1.36}\left(\mathrm{PO}_{4}\right)_{3}$, has been synthesized by means of solid-state reactions and characterized by single crystal X-ray diffraction.

\section{Structural commentary}

In the new isolated compound, either cobalt or iron atoms are distributed in the two octahedral sites while the phosphorus atoms are tetrahedrally coordinated, as shown in Fig. 1. The structure is built up from two edge-sharing [ $\left.(\mathrm{Co} 1 / \mathrm{Fe} 1) \mathrm{O}_{6}\right]$ octahedra, leading to the formation of $\left[(\mathrm{Co} 1 / \mathrm{Fe} 1)_{2} \mathrm{O}_{10}\right]$ dimers. Those dimers are connected by a common edge to $[(\mathrm{Fe} 2 /$ $\mathrm{Co} 2 \mathrm{CO}_{6}$ ] octahedra, forming an infinite chain (Fig. 2). The junction between these chains is ensured by sharing vertices with the $\mathrm{PO}_{4}$ tetrahedra so as to form an open layer perpendicular to [010] (Fig. 3). The three-dimensional framework resulting from the stacking of the sheets along the $b$-axis direction delimits channels parallel to [001] in which the $\mathrm{Ag}^{+}$ cations are accommodated, as shown in Fig. 4.

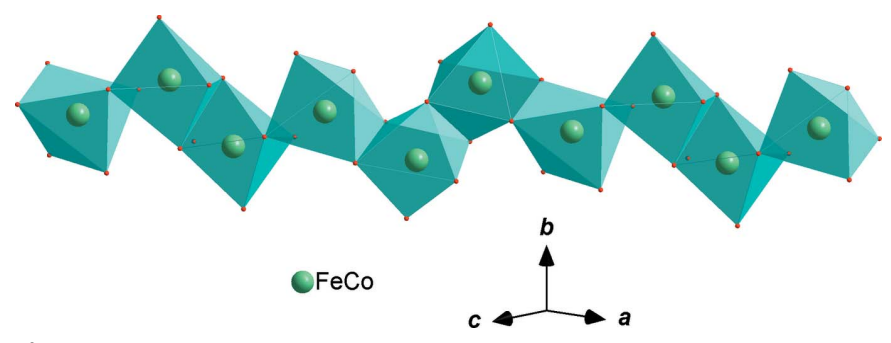

Figure 2

Edge-sharing $\left[(\mathrm{Fe} / \mathrm{Co}) \mathrm{O}_{6}\right]$ octahedra forming a layer parallel to [101].

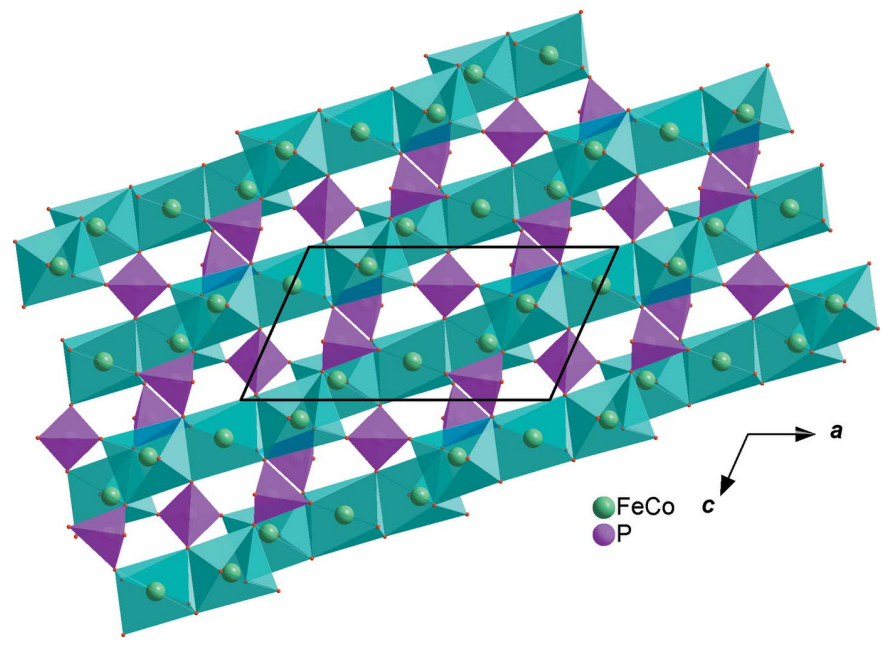

Figure 3

A view along [010], showing a layer resulting from the connection of chains via vertices of $\mathrm{PO}_{4}$ tetrahedra and $\left[\mathrm{FeO}_{6}\right]$ octahedra.

\section{Comparison with a related structure}

It is worth mentioning that the distribution of metallic cations observed in the case of the silver-cobalt-iron-based phosphate is not encountered in the sodium homologue. Hence, in the title silver-based phosphate, the octahedral $M 1$ site (Wyckoff position $8 f$ ) is occupied to $60 \%$ by $\mathrm{Fe} 1$ and to $40 \%$ by Co1. The octahedrally surrounded M2 site (Wyckoff position $4 e$ ) is essentially occupied by $\mathrm{Fe} 2$ atoms (43\%) along with a small amount of $\mathrm{Co} 2(7 \%)$. However, in the $\mathrm{Na}_{2} \mathrm{CO}_{2}$ $\mathrm{Fe}\left(\mathrm{PO}_{4}\right)_{3}$ phosphate, the $M 1$ and $M 2$ sites are entirely occupied by Fe and Co atoms, respectively. For the mixed sites, the occupancy rate was refined without any constraint. The results

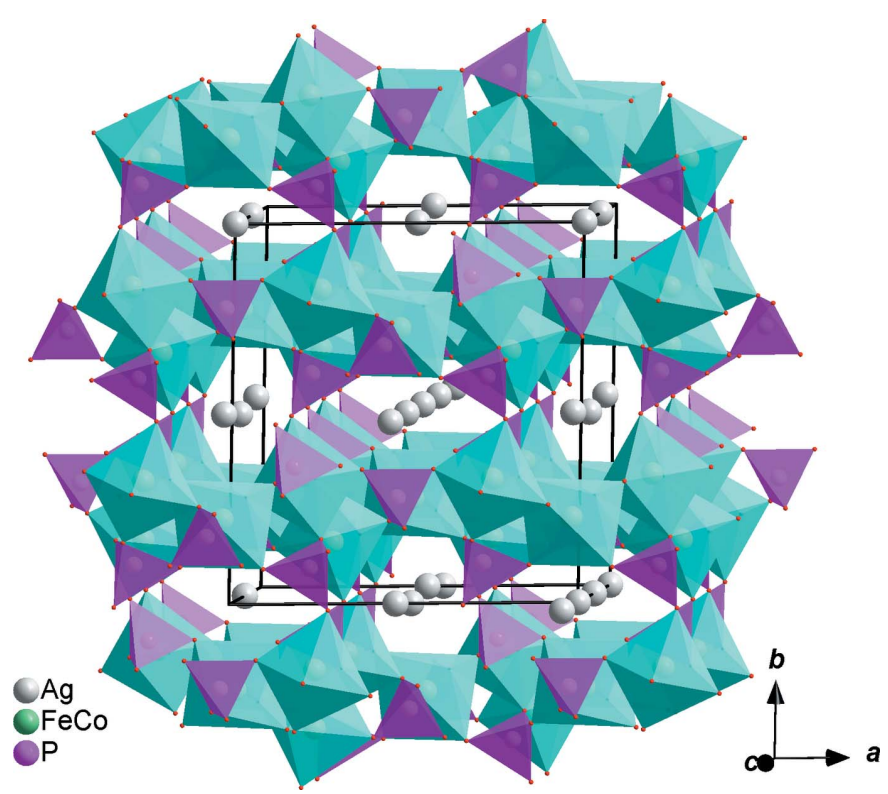

Figure 4

Polyhedral representation of $\mathrm{Ag}_{1.655} \mathrm{Co}_{1.64} \mathrm{Fe}_{1.36}\left(\mathrm{PO}_{4}\right)_{3}$, showing channels running along [001]. 
Table 1

Experimental details.

\begin{tabular}{|c|c|}
\hline \multicolumn{2}{|l|}{ Crystal data } \\
\hline Chemical formula & $\mathrm{Ag}_{1.655} \mathrm{Co}_{1.64} \mathrm{Fe}_{1.36}\left(\mathrm{PO}_{4}\right)_{3}$ \\
\hline$M_{\mathrm{r}}$ & 2544.10 \\
\hline Crystal system, space group & Monoclinic, $C 2 / c$ \\
\hline Temperature $(\mathrm{K})$ & 296 \\
\hline$a, b, c(\AA)$ & 11.8680 (3), 12.5514 (3), 6.4386 (2) \\
\hline$\beta\left(^{\circ}\right)$ & $114.012(1)$ \\
\hline$V\left(\AA^{6}\right)$ & $876.09(4)$ \\
\hline$Z$ & 1 \\
\hline Radiation type & Mo $K \alpha$ \\
\hline$\mu\left(\mathrm{mm}^{-1}\right)$ & 9.51 \\
\hline Crystal size $(\mathrm{mm})$ & $0.31 \times 0.26 \times 0.22$ \\
\hline \multicolumn{2}{|l|}{ Data collection } \\
\hline Diffractometer & Bruker X8 APEX \\
\hline Absorption correction & $\begin{array}{l}\text { Multi-scan (SADABS; Krause et } \\
\quad \text { al., 2015) }\end{array}$ \\
\hline$T_{\min }, T_{\max }$ & $0.066,0.124$ \\
\hline $\begin{array}{l}\text { No. of measured, independent and } \\
\text { observed }[I>2 \sigma(I)] \text { reflections }\end{array}$ & $13097,2137,2079$ \\
\hline$R_{\text {int }}$ & 0.030 \\
\hline$(\sin \theta / \lambda)_{\max }\left(\AA^{-1}\right)$ & 0.833 \\
\hline \multicolumn{2}{|l|}{ Refinement } \\
\hline$R\left[F^{2}>2 \sigma\left(F^{2}\right)\right], w R\left(F^{2}\right), S$ & $0.020,0.047,1.19$ \\
\hline No. of reflections & 2137 \\
\hline No. of parameters & 99 \\
\hline$\Delta \rho_{\max }, \Delta \rho_{\min }\left(\mathrm{e} \AA^{-3}\right)$ & $1.47,-0.92$ \\
\hline
\end{tabular}

Computer programs: APEX2 aand SAINT (Bruker, 2009), SHELXT2014 (Sheldrick, 2015a), SHELXL2014 (Sheldrick, 2015b), ORTEP-3 for Windows (Farrugia, 2012), DIAMOND (Brandenburg, 2006) and publCIF (Westrip, 2010).

of the refinements are in good agreement with the electrical neutrality of the compound and calculations of the bondvalence sums of the atoms in the structure (Brown \& Altermatt, 1985). Accordingly, in the silver-based phosphate, the cations at the $M 1$ site form double octahedra [ $\left.(\mathrm{Fe} 1 / \mathrm{Co} 1)_{2} \mathrm{O}_{10}\right]$ alternating with $\left[(\mathrm{Fe} 2 / \mathrm{Co} 2) \mathrm{O}_{6}\right]$ octahedra, while in the sodium homologue phosphate, the obtained $\left[\mathrm{Co}_{2} \mathrm{O}_{10}\right]$ double octahedra alternate with $\left[\mathrm{FeO}_{6}\right]$ octahedra (Fig. 4). Moreover, both the Ag1 and Ag2 atoms are located in channels, surrounded by eight oxygen atoms with $\mathrm{Ag} 1-\mathrm{O}$ bond lengths between 2.3320 (13) $\AA$ and 2.9176 (13) $\AA$, whereas Ag2-O bond lengths are in the range 2.4733 (13)-2.9035 (12) $\AA$. The structure of the title phosphate is isotypic to that of $\mathrm{Na}_{2} \mathrm{Co}_{2}$ $\mathrm{Fe}\left(\mathrm{PO}_{4}\right)_{3}$ (Bouraima et al., 2015) and $\mathrm{Na}_{1.67} \mathrm{Zn}_{1.67} \mathrm{Fe}_{1.33}\left(\mathrm{PO}_{4}\right)_{3}$ (Khmiyas et al., 2015).

\section{Synthesis and crystallization}

The title compound was isolated from solid-state reactions in air by mixing nitrates of silver, cobalt and iron with phosphoric acid. The various precursors are taken in the molar ratio Ag:Co:Fe:P = 2:2:1:3. The mixture was stirred at room temperature overnight. After different heat treatments in a platinum crucible at up to $873 \mathrm{~K}$, the reaction mixture was heated to the melting temperature of $1221 \mathrm{~K}$. The molten product was then cooled to room temperature at a rate of $5 \mathrm{~K} \mathrm{~h}^{-1}$. Brown homogeneous crystals corresponding to the title compound of a suitable size for X-ray diffraction were obtained.

\section{Refinement}

Crystal data, data collection and structure refinement details are summarized in Table 1 . The maximum and minimum residual electron densities in the final Fourier map are 0.68 and $0.55 \AA$ from Ag1 and Ag2, respectively.

\section{Acknowledgements}

The authors thank the Unit of Support for Technical and Scientific Research (UATRS, CNRST) for the X-ray measurements.

\section{Funding information}

Funding for this research was provided by: Mohammed V University, Rabat, Morocco.

\section{References}

Bouraima, A., Assani, A., Saadi, M., Makani, T. \& El Ammari, L. (2015). Acta Cryst. E71, 558-560.

Brandenburg, K. (2006). DIAMOND. Crystal Impact GbR, Bonn, Germany.

Brown, I. D. \& Altermatt, D. (1985). Acta Cryst. B41, 244-247.

Bruker (2009). APEX2 and SAINT. Bruker AXS Inc., Madison, Wisconsin, USA.

Farrugia, L. J. (2012). J. Appl. Cryst. 45, 849-854.

Hatert, F., Keller, P., Lissner, F., Antenucci, D. \& Fransolet, A.-M. (2000). Eur. J. Mineral. 12, 847-857.

Hatert, F., Long, G. J., Hautot, D., Fransolet, A.-M., Delwiche, J., Hubin-Franskin, M. J. \& Grandjean, F. (2004). Phys. Chem. Miner. 31, 487-506.

Khmiyas, J., Assani, A., Saadi, M. \& El Ammari, L. (2015). Acta Cryst. E71, 690-692.

Korzenski, M. B., Kolis, J. W. \& Long, G. J. (1999). J. Solid State Chem. 147, 390-398.

Krause, L., Herbst-Irmer, R., Sheldrick, G. M. \& Stalke, D. (2015). J. Appl. Cryst. 48, 3-10.

Moore, P. B. (1971). Am. Mineral. 56, 1955-1975.

Moore, P. B. \& Ito, J. (1979). Mineral. Mag. 43, 227-235.

Ould Saleck, A., Assani, A., Saadi, M., Mercier, C., Follet, C. \& El Ammari, L. (2015). Acta Cryst. E71, 813-815.

Sheldrick, G. M. (2015a). Acta Cryst. A71, 3-8.

Sheldrick, G. M. (2015b). Acta Cryst. C71, 3-8.

Trad, K., Carlier, D., Croguennec, L., Wattiaux, A., Ben Amara, M. \& Delmas, C. (2010). Chem. Mater. 22, 5554-5562.

Westrip, S. P. (2010). J. Appl. Cryst. 43, 920-925. 


\section{supporting information}

Acta Cryst. (2017). E73, 890-892 [https://doi.org/10.1107/S205698901700740X]

Crystal structure of a silver-, cobalt- and iron-based phosphate with an alluaudite-like structure: $\mathrm{Ag}_{1.655} \mathrm{Co}_{1.64} \mathrm{Fe}_{1.36}\left(\mathrm{PO}_{4}\right)_{3}$

Adam Bouraima, Thomas Makani, Abderrazzak Assani, Mohamed Saadi and Lahcen El Ammari

Computing details

Data collection: APEX2 (Bruker, 2009); cell refinement: SAINT (Bruker, 2009); data reduction: SAINT (Bruker, 2009); program(s) used to solve structure: SHELXT2014 (Sheldrick, 2015a); program(s) used to refine structure: SHELXL2014 (Sheldrick, 2015b); molecular graphics: ORTEP-3 for Windows (Farrugia, 2012) and DIAMOND (Brandenburg, 2006); software used to prepare material for publication: publCIF (Westrip, 2010).

Silver cobalt iron tris(orthophosphate)

Crystal data

$\mathrm{Ag}_{1.655} \mathrm{Co}_{1.64} \mathrm{Fe}_{1.36}\left(\mathrm{PO}_{4}\right)_{3}$

$M_{r}=2544.10$

Monoclinic, $C 2 / c$

$a=11.8680(3) \AA$

$b=12.5514(3) \AA$

$c=6.4386(2) \AA$

$\beta=114.012(1)^{\circ}$

$V=876.09(4) \AA^{3}$

$Z=1$

\section{Data collection}

Bruker X8 APEX diffractometer

Radiation source: fine-focus sealed tube Graphite monochromator $\varphi$ and $\omega$ scans

Absorption correction: multi-scan

(SADABS; Krause et al., 2015)

$T_{\min }=0.066, T_{\max }=0.124$

Refinement

Refinement on $F^{2}$

Least-squares matrix: full

$R\left[F^{2}>2 \sigma\left(F^{2}\right)\right]=0.020$

$w R\left(F^{2}\right)=0.047$

$S=1.19$

2137 reflections

99 parameters

0 restraints
$F(000)=1194$

$D_{\mathrm{x}}=4.822 \mathrm{Mg} \mathrm{m}^{-3}$

Mo $K \alpha$ radiation, $\lambda=0.71073 \AA$

Cell parameters from 2137 reflections

$\theta=3.3-36.3^{\circ}$

$\mu=9.51 \mathrm{~mm}^{-1}$

$T=296 \mathrm{~K}$

Block, brown

$0.31 \times 0.26 \times 0.22 \mathrm{~mm}$

13097 measured reflections

2137 independent reflections

2079 reflections with $I>2 \sigma(I)$

$R_{\text {int }}=0.030$

$\theta_{\max }=36.3^{\circ}, \theta_{\min }=3.3^{\circ}$

$h=-19 \rightarrow 19$

$k=-20 \rightarrow 20$

$l=-10 \rightarrow 10$

$w=1 /\left[\sigma^{2}\left(F_{\mathrm{o}}^{2}\right)+(0.0121 P)^{2}+3.2851 P\right]$

where $P=\left(F_{\mathrm{o}}^{2}+2 F_{\mathrm{c}}^{2}\right) / 3$

$(\Delta / \sigma)_{\max }=0.001$

$\Delta \rho_{\max }=1.47 \mathrm{e} \AA^{-3}$

$\Delta \rho_{\min }=-0.92$ e $\AA^{-3}$

Extinction correction: SHELXL2014

(Sheldrick, 2015b),

$\mathrm{Fc}^{*}=\mathrm{kFc}\left[1+0.001 \mathrm{xFc}^{2} \lambda^{3} / \sin (2 \theta)\right]^{-1 / 4}$

Extinction coefficient: 0.00102 (10) 


\section{Special details}

Geometry. All esds (except the esd in the dihedral angle between two 1.s. planes) are estimated using the full covariance matrix. The cell esds are taken into account individually in the estimation of esds in distances, angles and torsion angles; correlations between esds in cell parameters are only used when they are defined by crystal symmetry. An approximate (isotropic) treatment of cell esds is used for estimating esds involving l.s. planes.

Fractional atomic coordinates and isotropic or equivalent isotropic displacement parameters $\left(\hat{A}^{2}\right)$

\begin{tabular}{llllll}
\hline & $x$ & $y$ & $z$ & $U_{\text {iso }} * U_{\text {eq }}$ & Occ. $(<1)$ \\
\hline Ag1 & 0.5000 & 0.5000 & 0.5000 & $0.01952(7)$ & $0.885(2)$ \\
$\mathrm{Ag} 2$ & 1.0000 & $0.48916(3)$ & 0.7500 & $0.02408(10)$ & $0.7688(19)$ \\
$\mathrm{Fe} 1$ & $0.78227(2)$ & $0.34311(2)$ & $0.37115(3)$ & $0.00565(6)$ & $0.61(4)$ \\
$\mathrm{Co} 1$ & $0.78227(2)$ & $0.34311(2)$ & $0.37115(3)$ & $0.00565(6)$ & $0.39(4)$ \\
$\mathrm{Fe} 2$ & 1.0000 & $0.76503(2)$ & 0.7500 & $0.00714(8)$ & $0.14(5)$ \\
$\mathrm{Co} 2$ & 1.0000 & $0.76503(2)$ & 0.7500 & $0.00714(8)$ & $0.86(5)$ \\
P1 & $0.76272(3)$ & $0.61138(3)$ & $0.37428(6)$ & $0.00502(8)$ & \\
P2 & 0.5000 & $0.28909(4)$ & 0.2500 & $0.00535(10)$ & \\
O1 & $0.77807(11)$ & $0.67841(10)$ & $0.18620(19)$ & $0.00908(19)$ & \\
O2 & $0.81856(12)$ & $0.49999(9)$ & $0.3820(2)$ & $0.0112(2)$ & \\
O3 & $0.62598(11)$ & $0.60711(11)$ & $0.3280(2)$ & $0.0135(2)$ & \\
O4 & $0.83676(12)$ & $0.66524(9)$ & $0.60788(19)$ & $0.00917(19)$ & \\
O5 & $0.45841(10)$ & $0.21873(10)$ & $0.03381(18)$ & $0.00821(19)$ & \\
O6 & $0.60284(12)$ & $0.36401(10)$ & $0.2530(2)$ & $0.0122(2)$ & \\
& & & & &
\end{tabular}

Atomic displacement parameters $\left(\AA^{2}\right)$

\begin{tabular}{lllllll}
\hline & $U^{11}$ & $U^{22}$ & $U^{33}$ & $U^{12}$ & $U^{13}$ & $U^{23}$ \\
\hline Ag1 & $0.02917(14)$ & $0.00879(9)$ & $0.01123(10)$ & $-0.00389(7)$ & $-0.00138(8)$ & $-0.00128(6)$ \\
Ag2 & $0.01069(12)$ & $0.02795(16)$ & $0.02519(15)$ & 0.000 & $-0.00133(10)$ & 0.000 \\
Fe1 & $0.00485(9)$ & $0.00657(9)$ & $0.00575(9)$ & $0.00037(6)$ & $0.00240(6)$ & $0.00058(6)$ \\
Co1 & $0.00485(9)$ & $0.00657(9)$ & $0.00575(9)$ & $0.00037(6)$ & $0.00240(6)$ & $0.00058(6)$ \\
Fe2 & $0.00620(12)$ & $0.00833(13)$ & $0.00796(13)$ & 0.000 & $0.00398(10)$ & 0.000 \\
Co2 & $0.00620(12)$ & $0.00833(13)$ & $0.00796(13)$ & 0.000 & $0.00398(10)$ & 0.000 \\
P1 & $0.00488(15)$ & $0.00490(15)$ & $0.00521(15)$ & $0.00006(11)$ & $0.00198(11)$ & $0.00022(10)$ \\
P2 & $0.00397(19)$ & $0.0071(2)$ & $0.00466(19)$ & 0.000 & $0.00138(16)$ & 0.000 \\
O1 & $0.0101(5)$ & $0.0112(5)$ & $0.0062(4)$ & $-0.0001(4)$ & $0.0036(4)$ & $0.0020(3)$ \\
O2 & $0.0118(5)$ & $0.0070(4)$ & $0.0144(5)$ & $0.0024(4)$ & $0.0048(4)$ & $-0.0013(4)$ \\
O3 & $0.0067(4)$ & $0.0125(5)$ & $0.0221(6)$ & $0.0007(4)$ & $0.0066(4)$ & $0.0031(4)$ \\
O4 & $0.0127(5)$ & $0.0083(4)$ & $0.0062(4)$ & $-0.0011(4)$ & $0.0034(4)$ & $-0.0013(3)$ \\
O5 & $0.0064(4)$ & $0.0124(5)$ & $0.0053(4)$ & $-0.0011(4)$ & $0.0018(3)$ & $-0.0017(3)$ \\
O6 & $0.0085(5)$ & $0.0119(5)$ & $0.0166(5)$ & $-0.0036(4)$ & $0.0054(4)$ & $0.0007(4)$ \\
& & & & & &
\end{tabular}

Geometric parameters $\left(\AA,{ }^{\circ}\right)$

\begin{tabular}{llll}
\hline $\mathrm{Ag} 1-\mathrm{O}^{\mathrm{i}}$ & $2.3320(13)$ & $\mathrm{Fe} 1-\mathrm{O} 4^{\text {viii }}$ & $2.0481(12)$ \\
$\mathrm{Ag} 1-\mathrm{O} 6^{\mathrm{ii}}$ & $2.3320(13)$ & $\mathrm{Fe} 1-\mathrm{O} 1^{\mathrm{i}}$ & $2.0669(11)$ \\
$\mathrm{Ag} 1-\mathrm{O} 3^{\mathrm{i}}$ & $2.4356(14)$ & $\mathrm{Fe} 1-\mathrm{O} 5^{\mathrm{vi}}$ & $2.0705(12)$ \\
$\mathrm{Ag} 1-\mathrm{O} 3^{\text {ii }}$ & $2.4356(14)$ & $\mathrm{Fe} 1-\mathrm{O} 1^{\mathrm{ix}}$ & $2.1695(12)$
\end{tabular}




\begin{tabular}{|c|c|c|c|}
\hline $\mathrm{Ag} 1-\mathrm{O} 3^{\mathrm{iii}}$ & $2.5724(13)$ & $\mathrm{Fe} 2-\mathrm{O}^{\mathrm{x}}$ & $2.1099(13)$ \\
\hline $\mathrm{Ag} 1-\mathrm{O} 3$ & $2.5725(13)$ & $\mathrm{Fe} 2-\mathrm{O} 3^{\mathrm{xi}}$ & $2.1099(13)$ \\
\hline $\mathrm{Ag} 1-\mathrm{O} 6^{\mathrm{iii}}$ & $2.9176(13)$ & $\mathrm{Fe} 2-\mathrm{O}^{\mathrm{xii}}$ & $2.1575(11)$ \\
\hline $\mathrm{Ag} 1-\mathrm{O} 6$ & $2.9176(13)$ & $\mathrm{Fe} 2-\mathrm{O} 5^{\mathrm{xiii}}$ & $2.1575(11)$ \\
\hline $\mathrm{Ag} 2-\mathrm{O} 2^{\mathrm{iv}}$ & $2.4733(13)$ & $\mathrm{Fe} 2-\mathrm{O} 4^{\text {iv }}$ & $2.1717(12)$ \\
\hline $\mathrm{Ag} 2-\mathrm{O} 2$ & $2.4733(13)$ & $\mathrm{Fe} 2-\mathrm{O} 4$ & $2.1717(12)$ \\
\hline $\mathrm{Ag} 2-\mathrm{O} 2^{\mathrm{i}}$ & $2.6204(13)$ & $\mathrm{P} 1-\mathrm{O} 3$ & $1.5270(13)$ \\
\hline $\mathrm{Ag} 2-\mathrm{O} 2^{\mathrm{v}}$ & $2.6204(13)$ & $\mathrm{P} 1-\mathrm{O} 2$ & $1.5393(12)$ \\
\hline $\mathrm{Ag} 2-\mathrm{O} 4$ & $2.8341(12)$ & $\mathrm{P} 1-\mathrm{O} 1$ & $1.5451(12)$ \\
\hline $\mathrm{Ag} 2-\mathrm{O} 4^{\mathrm{iv}}$ & $2.8341(12)$ & $\mathrm{P} 1-\mathrm{O} 4$ & $1.5543(12)$ \\
\hline $\mathrm{Ag} 2-\mathrm{O} 5^{\mathrm{vi}}$ & $2.9035(13)$ & $\mathrm{P} 2-\mathrm{O} 6^{\mathrm{ii}}$ & $1.5346(13)$ \\
\hline $\mathrm{Ag} 2-\mathrm{O} 5^{\mathrm{vii}}$ & $2.9035(12)$ & $\mathrm{P} 2-\mathrm{O} 6$ & $1.5346(13)$ \\
\hline $\mathrm{Fe} 1-\mathrm{O} 6$ & $1.9656(13)$ & $\mathrm{P} 2-\mathrm{O} 5^{\mathrm{ii}}$ & $1.5498(12)$ \\
\hline $\mathrm{Fe} 1-\mathrm{O} 2$ & $2.0108(12)$ & $\mathrm{P} 2-\mathrm{O} 5$ & $1.5498(12)$ \\
\hline $\mathrm{O} 6^{\mathrm{i}}-\mathrm{Ag} 1-\mathrm{O} 6^{\mathrm{ii}}$ & $180.00(4)$ & $\mathrm{O} 2-\mathrm{Ag} 2-\mathrm{O} 1^{\mathrm{i}}$ & $64.62(4)$ \\
\hline $\mathrm{O} 6^{\mathrm{i}}-\mathrm{Ag} 1-\mathrm{O} 3^{\mathrm{i}}$ & $80.58(4)$ & $\mathrm{O} 2^{\mathrm{i}}-\mathrm{Ag} 2-\mathrm{O} 1^{\mathrm{i}}$ & $49.21(3)$ \\
\hline $\mathrm{O} 6^{\mathrm{ii}}-\mathrm{Ag} 1-\mathrm{O}^{\mathrm{i}}$ & $99.42(4)$ & $\mathrm{O} 2^{\mathrm{v}}-\mathrm{Ag} 2-\mathrm{O} 1^{\mathrm{i}}$ & $136.08(3)$ \\
\hline $\mathrm{O}^{\mathrm{i}}-\mathrm{Ag} 1-\mathrm{O} 3^{\mathrm{ii}}$ & $99.42(4)$ & $\mathrm{O} 4-\mathrm{Ag} 2-\mathrm{O} 1^{\mathrm{i}}$ & $92.94(3)$ \\
\hline $\mathrm{O} 6^{\mathrm{ii}}-\mathrm{Ag} 1-\mathrm{O} 3^{\mathrm{ii}}$ & $80.58(4)$ & $\mathrm{O} 4^{\mathrm{iv}}-\mathrm{Ag} 2-\mathrm{O} 1^{\mathrm{i}}$ & $162.54(3)$ \\
\hline $\mathrm{O} 3^{\mathrm{i}}-\mathrm{Ag} 1-\mathrm{O} 3^{\mathrm{ii}}$ & 180.0 & $\mathrm{O}^{\mathrm{vi}}-\mathrm{Ag} 2-\mathrm{O} 1^{\mathrm{i}}$ & $52.36(3)$ \\
\hline $\mathrm{O} 6^{\mathrm{i}}-\mathrm{Ag} 1-\mathrm{O}^{\mathrm{iii}}$ & $108.21(5)$ & $\mathrm{O}^{\mathrm{vii}}-\mathrm{Ag} 2-\mathrm{O} 1^{\mathrm{i}}$ & $56.78(3)$ \\
\hline $\mathrm{O} 6^{\mathrm{ii}}-\mathrm{Ag} 1-\mathrm{O}^{\mathrm{iii}}$ & $71.79(5)$ & $\mathrm{O} 2^{\mathrm{iv}}-\mathrm{Ag} 2-\mathrm{O}^{\mathrm{v}}$ & $64.62(4)$ \\
\hline $\mathrm{O} 3^{\mathrm{i}}-\mathrm{Ag} 1-\mathrm{O} 3^{\mathrm{iii}}$ & $66.27(5)$ & $\mathrm{O} 2-\mathrm{Ag} 2-\mathrm{O}^{\mathrm{v}}$ & $119.96(4)$ \\
\hline $\mathrm{O}{ }^{\mathrm{ii}}-\mathrm{Ag} 1-\mathrm{O}^{\mathrm{iii}}$ & $113.73(5)$ & $\mathrm{O} 2^{\mathrm{i}}-\mathrm{Ag} 2-\mathrm{O}^{\mathrm{v}}$ & $136.08(3)$ \\
\hline $\mathrm{O} 6 \mathrm{i}-\mathrm{Ag} 1-\mathrm{O} 3$ & $71.79(5)$ & $\mathrm{O} 2^{\mathrm{v}}-\mathrm{Ag} 2-\mathrm{O} 1^{\mathrm{v}}$ & $49.21(3)$ \\
\hline $\mathrm{O} 6{ }^{\mathrm{ii}}-\mathrm{Ag} 1-\mathrm{O} 3$ & $108.21(5)$ & $\mathrm{O} 4-\mathrm{Ag} 2-\mathrm{O}^{\mathrm{v}}$ & $162.54(3)$ \\
\hline $\mathrm{O} 33^{\mathrm{i}}-\mathrm{Ag} 1-\mathrm{O} 3$ & $113.73(5)$ & $\mathrm{O} 4^{\mathrm{iv}}-\mathrm{Ag} 2-\mathrm{O} 1^{\mathrm{v}}$ & $92.94(3)$ \\
\hline $\mathrm{O} 3{ }^{\mathrm{ii}}-\mathrm{Ag} 1-\mathrm{O} 3$ & $66.27(5)$ & $\mathrm{O}^{\mathrm{vi}}-\mathrm{Ag} 2-\mathrm{O}^{\mathrm{v}}$ & $56.78(3)$ \\
\hline $\mathrm{O} 3^{\mathrm{iii}-\mathrm{Ag} 1-\mathrm{O} 3}$ & 180.0 & $\mathrm{O}^{\mathrm{vii}}-\mathrm{Ag} 2-\mathrm{O} 1^{\mathrm{v}}$ & $52.36(3)$ \\
\hline $\mathrm{O} 6^{\mathrm{i}}-\mathrm{Ag} 1-\mathrm{O}^{\mathrm{iii}}$ & $53.64(5)$ & $\mathrm{O} 6-\mathrm{Fe} 1-\mathrm{O} 2$ & $93.77(5)$ \\
\hline $\mathrm{O} 6^{\mathrm{ii}}-\mathrm{Ag} 1-\mathrm{O}^{\mathrm{iii}}$ & $126.36(5)$ & $\mathrm{O} 6-\mathrm{Fe} 1-\mathrm{O} 4^{\mathrm{viii}}$ & $110.10(5)$ \\
\hline $\mathrm{O} 3^{\mathrm{i}}-\mathrm{Ag} 1-\mathrm{O}^{\mathrm{iii}}$ & $95.49(4)$ & $\mathrm{O} 2-\mathrm{Fe} 1-\mathrm{O} 4^{\text {viii }}$ & $86.76(5)$ \\
\hline $\mathrm{O}^{\mathrm{ii}}-\mathrm{Ag} 1-\mathrm{O}^{\mathrm{iii}}$ & $84.51(4)$ & $\mathrm{O} 6-\mathrm{Fe} 1-\mathrm{O} 1^{\mathrm{i}}$ & $86.70(5)$ \\
\hline $\mathrm{O} 3^{\mathrm{iii}}-\mathrm{Ag} 1-\mathrm{O} 6^{\mathrm{iii}}$ & $68.02(4)$ & $\mathrm{O} 2-\mathrm{Fe} 1-\mathrm{O} 1^{\mathrm{i}}$ & $100.62(5)$ \\
\hline $\mathrm{O} 3-\mathrm{Ag} 1-\mathrm{O} 6^{\mathrm{iii}}$ & $111.98(4)$ & $\mathrm{O} 4^{\mathrm{viii}}-\mathrm{Fe} 1-\mathrm{O} 1^{\mathrm{i}}$ & $161.33(5)$ \\
\hline $\mathrm{O} 6 \mathrm{i}-\mathrm{Ag} 1-\mathrm{O} 6$ & $126.36(5)$ & $\mathrm{O} 6-\mathrm{Fe} 1-\mathrm{O}^{\mathrm{vi}}$ & $163.25(5)$ \\
\hline $\mathrm{O} 6 \mathrm{i}^{\mathrm{ii}}-\mathrm{Ag} 1-\mathrm{O} 6$ & $53.64(5)$ & $\mathrm{O} 2-\mathrm{Fe} 1-\mathrm{O} 5^{\mathrm{vi}}$ & $101.04(5)$ \\
\hline $\mathrm{O} 3{ }^{\mathrm{i}}-\mathrm{Ag} 1-\mathrm{O} 6$ & $84.51(4)$ & $\mathrm{O} 4^{\mathrm{viii}}-\mathrm{Fe} 1-\mathrm{O} 5^{\mathrm{vi}}$ & $78.79(5)$ \\
\hline $\mathrm{O}^{\mathrm{ii}}-\mathrm{Ag} 1-\mathrm{O} 6$ & $95.49(4)$ & $\mathrm{O} 1^{\mathrm{i}}-\mathrm{Fe} 1-\mathrm{O}^{\mathrm{vi}}$ & $82.95(5)$ \\
\hline $\mathrm{O} 3^{\mathrm{iii}-\mathrm{Ag} 1-\mathrm{O} 6}$ & $111.98(4)$ & $\mathrm{O} 6-\mathrm{Fe} 1-\mathrm{O} 1^{\mathrm{ix}}$ & $80.26(5)$ \\
\hline $\mathrm{O} 3-\mathrm{Ag} 1-\mathrm{O} 6$ & $68.02(4)$ & $\mathrm{O} 2-\mathrm{Fe} 1-\mathrm{O} 1^{\mathrm{ix}}$ & $171.95(5)$ \\
\hline $\mathrm{O}^{\mathrm{iii}}-\mathrm{Ag} 1-\mathrm{O} 6$ & 180.0 & 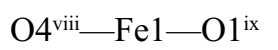 & $90.22(4)$ \\
\hline $\mathrm{O} 2^{\mathrm{iv}}-\mathrm{Ag} 2-\mathrm{O} 2$ & $173.70(6)$ & 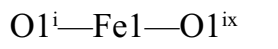 & $84.52(5)$ \\
\hline $\mathrm{O} 2^{\mathrm{iv}}-\mathrm{Ag} 2-\mathrm{O} 2^{\mathrm{i}}$ & $101.33(4)$ & $\mathrm{O} 5^{\mathrm{vi}}-\mathrm{Fe} 1-\mathrm{O} 1^{\mathrm{ix}}$ & $85.66(5)$ \\
\hline $\mathrm{O} 2-\mathrm{Ag} 2-\mathrm{O} 2^{\mathrm{i}}$ & $78.34(4)$ & $\mathrm{O} 3^{\mathrm{x}}-\mathrm{Fe} 2-\mathrm{O}^{{ }^{\mathrm{xi}}}$ & $80.97(7)$ \\
\hline $\mathrm{O} 2^{\mathrm{iv}}-\mathrm{Ag} 2-\mathrm{O} 2^{\mathrm{v}}$ & $78.34(4)$ & $\mathrm{O} 3^{\mathrm{x}}-\mathrm{Fe} 2-\mathrm{O} 5^{\mathrm{xii}}$ & $91.27(5)$ \\
\hline $\mathrm{O} 2-\mathrm{Ag} 2-\mathrm{O}^{\mathrm{v}}$ & $101.33(4)$ & $\mathrm{O} 3^{\mathrm{xi}}-\mathrm{Fe} 2-\mathrm{O} 5^{\mathrm{xii}}$ & $112.81(5)$ \\
\hline
\end{tabular}




\begin{tabular}{|c|c|c|c|}
\hline $\mathrm{O} 2^{\mathrm{i}}-\mathrm{Ag} 2-\mathrm{O} 2^{\mathrm{v}}$ & $174.04(5)$ & $\mathrm{O} 3^{\mathrm{x}}-\mathrm{Fe} 2-\mathrm{O}^{\mathrm{xiii}}$ & $112.81(5)$ \\
\hline $\mathrm{O} 2^{\mathrm{iv}}-\mathrm{Ag} 2-\mathrm{O} 4$ & $118.73(4)$ & $\mathrm{O} 3^{\mathrm{xi}}-\mathrm{Fe} 2-\mathrm{O} 5^{\mathrm{xii}}$ & $91.27(5)$ \\
\hline $\mathrm{O} 2-\mathrm{Ag} 2-\mathrm{O} 4$ & $55.50(4)$ & $\mathrm{O} 5^{\mathrm{xii}}-\mathrm{Fe} 2-\mathrm{O} 5^{\mathrm{xiii}}$ & $148.74(7)$ \\
\hline $\mathrm{O} 2 \mathrm{i}-\mathrm{Ag} 2-\mathrm{O} 4$ & $61.33(4)$ & $\mathrm{O} 3^{\mathrm{x}}-\mathrm{Fe} 2-\mathrm{O} 4^{\mathrm{iv}}$ & $85.08(5)$ \\
\hline $\mathrm{O} 2^{\mathrm{v}}-\mathrm{Ag} 2-\mathrm{O} 4$ & $113.50(4)$ & $\mathrm{O} 3^{\mathrm{xi}}-\mathrm{Fe} 2-\mathrm{O} 4^{\mathrm{iv}}$ & $164.39(5)$ \\
\hline $\mathrm{O} 2^{\mathrm{iv}}-\mathrm{Ag} 2-\mathrm{O} 4^{\mathrm{iv}}$ & $55.50(4)$ & $\mathrm{O} 5^{\mathrm{xii}}-\mathrm{Fe} 2-\mathrm{O} 4^{\mathrm{iv}}$ & $74.29(4)$ \\
\hline $\mathrm{O} 2-\mathrm{Ag} 2-\mathrm{O} 4^{\mathrm{iv}}$ & $118.73(4)$ & $\mathrm{O} 5^{\mathrm{xiii}}-\mathrm{Fe} 2-\mathrm{O} 4^{\mathrm{iv}}$ & $87.71(4)$ \\
\hline $\mathrm{O} 2^{\mathrm{i}}-\mathrm{Ag} 2-\mathrm{O}^{\mathrm{iv}}$ & $113.50(4)$ & $\mathrm{O}^{2}-\mathrm{Fe} 2-\mathrm{O} 4$ & $164.39(5)$ \\
\hline $\mathrm{O} 2^{\mathrm{v}}-\mathrm{Ag} 2-\mathrm{O} 4^{\mathrm{iv}}$ & $61.33(4)$ & $\mathrm{O} 3^{\mathrm{xi}-\mathrm{Fe} 2-\mathrm{O} 4}$ & $85.08(5)$ \\
\hline $\mathrm{O} 4-\mathrm{Ag} 2-\mathrm{O}_{4}^{\mathrm{iv}}$ & $77.51(5)$ & $\mathrm{O} 5^{\mathrm{xii}}-\mathrm{Fe} 2-\mathrm{O} 4$ & $87.71(4)$ \\
\hline $\mathrm{O} 2^{\mathrm{iv}}-\mathrm{Ag} 2-\mathrm{O}^{\mathrm{vi}}$ & $114.87(4)$ & $\mathrm{O} 5^{\text {xiii_- }} \mathrm{Fe} 2-\mathrm{O} 4$ & $74.29(4)$ \\
\hline $\mathrm{O} 2-\mathrm{Ag} 2-\mathrm{O}^{\mathrm{vi}}$ & $71.23(4)$ & $\mathrm{O} 4^{\mathrm{iv}}-\mathrm{Fe} 2-\mathrm{O} 4$ & $109.56(7)$ \\
\hline $\mathrm{O} 2^{\mathrm{i}}-\mathrm{Ag} 2-\mathrm{O}^{\mathrm{vi}}$ & $101.56(4)$ & $\mathrm{O} 3-\mathrm{P} 1-\mathrm{O} 2$ & $112.57(7)$ \\
\hline $\mathrm{O} 2^{\mathrm{v}}-\mathrm{Ag} 2-\mathrm{O}^{\mathrm{vi}}$ & $83.86(4)$ & $\mathrm{O} 3-\mathrm{P} 1-\mathrm{O} 1$ & $108.72(7)$ \\
\hline $\mathrm{O} 4-\mathrm{Ag} 2-\mathrm{O}^{\mathrm{vi}}$ & $125.84(3)$ & $\mathrm{O} 2-\mathrm{P} 1-\mathrm{O} 1$ & 109.47 (7) \\
\hline $\mathrm{O} 4^{\mathrm{iv}}-\mathrm{Ag} 2-\mathrm{O} 5^{\mathrm{vi}}$ & $144.70(3)$ & $\mathrm{O} 3-\mathrm{P} 1-\mathrm{O} 4$ & $109.98(7)$ \\
\hline $\mathrm{O} 2^{\mathrm{iv}}-\mathrm{Ag} 2-\mathrm{O} 5^{\mathrm{vii}}$ & $71.23(4)$ & $\mathrm{O} 2-\mathrm{P} 1-\mathrm{O} 4$ & $107.31(7)$ \\
\hline $\mathrm{O} 2-\mathrm{Ag} 2-\mathrm{O}^{\mathrm{vii}}$ & $114.87(4)$ & $\mathrm{O} 1-\mathrm{P} 1-\mathrm{O} 4$ & $108.73(7)$ \\
\hline $\mathrm{O} 2^{\mathrm{i}}-\mathrm{Ag} 2-\mathrm{O}^{\mathrm{vii}}$ & $83.86(4)$ & $\mathrm{O} 6{ }^{\mathrm{ii}}-\mathrm{P} 2-\mathrm{O} 6$ & $104.43(10)$ \\
\hline $\mathrm{O} 2^{\mathrm{v}}-\mathrm{Ag} 2-\mathrm{O} 5^{\mathrm{vii}}$ & $101.56(4)$ & $\mathrm{O} 6^{\mathrm{ii}}-\mathrm{P} 2-\mathrm{O}^{\mathrm{ii}}$ & $108.75(7)$ \\
\hline $\mathrm{O} 4-\mathrm{Ag} 2-\mathrm{O}^{\mathrm{vii}}$ & $144.70(3)$ & $\mathrm{O} 6-\mathrm{P} 2-\mathrm{O} 5^{\mathrm{ii}}$ & $112.14(7)$ \\
\hline $\mathrm{O} 4^{\mathrm{iv}}-\mathrm{Ag} 2-\mathrm{O} 5^{\mathrm{vii}}$ & $125.84(3)$ & $\mathrm{O} 6{ }^{\mathrm{ii}}-\mathrm{P} 2-\mathrm{O} 5$ & $112.14(7)$ \\
\hline $\mathrm{O} 5^{\mathrm{vi}}-\mathrm{Ag} 2-\mathrm{O} 5^{\mathrm{vii}}$ & $52.03(4)$ & $\mathrm{O} 6-\mathrm{P} 2-\mathrm{O} 5$ & $108.75(7)$ \\
\hline $\mathrm{O} 2^{\mathrm{iv}}-\mathrm{Ag} 2-\mathrm{O} 1^{\mathrm{i}}$ & $119.96(4)$ & $\mathrm{O} 5^{\mathrm{ii}}-\mathrm{P} 2-\mathrm{O} 5$ & $110.52(9)$ \\
\hline
\end{tabular}

Symmetry codes: (i) $x,-y+1, z+1 / 2$; (ii) $-x+1, y,-z+1 / 2$; (iii) $-x+1,-y+1,-z+1$; (iv) $-x+2, y,-z+3 / 2$; (v) $-x+2,-y+1,-z+1$; (vi) $x+1 / 2,-y+1 / 2, z+1 / 2$; (vii) $-x+3 / 2,-y+1 / 2,-z+1$; (viii) $x,-y+1, z-1 / 2$; (ix) $-x+3 / 2, y-1 / 2,-z+1 / 2$; (x) $x+1 / 2,-y+3 / 2, z+1 / 2$; (xi) $-x+3 / 2,-y+3 / 2,-z+1$; (xii) $-x+3 / 2, y+1 / 2$, $-z+1 / 2$; (xiii) $x+1 / 2, y+1 / 2, z+1$. 